\title{
Abraham Flexner a-t-il provoqué la création du JAMC ?
}

\author{
Jacalyn Duffin MD PhD
}

$\mathrm{L}$

'Association médicale canadienne (AMC) a été fondée en 1867, mais son journal n'est apparu que 44 ans plus tard. Pourquoi en 1911 ?

Des chercheurs en ont déjà documenté les facteurs précipitants : le souhait de longue date de l'AMC de fonder un journal national, la disponibilité d'un rédacteur en chef talentueux en la personne d'Andrew Macphail et la disparition volontaire de deux périodiques régionaux ${ }^{1}$. Je soutiens ici que le Journal de l'Association médicale canadienne (JAMC) a vu le jour en 1911 comme réaction nationaliste au rapport Flexner de 1910 (Medical education in the United States and Canada: a report to the Carnegie Foundation for the Advancement of Teaching) $)^{2}$. Le volume $1 \mathrm{du} J A M C$ contient une abondance de données probantes.

En 1909, préoccupé par la qualité inégale et le manque de connaissances scientifiques à jour dans les facultés de médecine, le conseil de l'éducation médicale de l'American Medical Association invita la Carnegie Foundation for the Advancement of Teaching à enquêter sur l'éducation médicale en Amérique du Nord. En l'espace de 18 mois, Abraham Flexner, éducateur choisi personnellement par le président de la Fondation Carnegie, rendit visite à 155 facultés de médecine en Amérique du Nord. Selon lui et selon le conseil, les étudiants devaient être sélectionnés en fonction de normes d'admission rigoureuses, apprendre les sciences fondamentales qui leur seraient enseignées par des professeurs universitaires à temps plein dans des laboratoires bien équipés et avoir libre accès aux cas cliniques dans les hôpitaux. Son étalon-or — et le seul élément positif à ressortir de l'enquête — fut l'Université Johns Hopkins ${ }^{3}$.

En 1920, une décennie après la publication du rapport Flexner, le nombre de facultés de médecine aux États-Unis était tombé de 147 à 85 et continuait à diminuer. Les établissements mal financés, surtout ceux qui formaient des femmes et des Afro-américains, avaient souffert le plus ${ }^{4}$. Alors que certaines de ces fermetures avaient peutêtre été rendues inévitables par la montée des coûts administratifs, d'autres écoles disparurent à la suite de fusions ${ }^{5}$. Que Flexner ait directement causé ces changements ou les ait simplement préludés, son rapport servit de plan directeur pour l'éducation médicale au cours des décennies qui suivirent.

Des années plus tard, Flexner se vanta de sa capacité à saisir l'essentiel d'un établissement en quelques heures : il lui suffisait de feuilleter rapidement les dossiers d'admission, de se balader dans les services, de humer l'atmosphère, de tromper ses hôtes et même d'acheter les concierges pour leur faire ouvrir des portes verrouillées ${ }^{6}$. "Caustique, arrogant et intransigeant », Flexner se fit des ennemis partout. « Agressifs, clairs et francs », ses jugements ne manquaient certes pas de visibilité?

Les résultats des trois brèves visites de Flexner dans les huit facultés de médecine du Canada en mars, mai et octobre 1909 occupaient sept pages à la fin de son rapport, qui fut rendu public en avril $1910^{2}$. Même si elles n'étaient pas parfaites, les facultés de Toronto et de McGill étaient « excellentes » et vouées à l'amélioration. Celle du Manitoba était bonne, ses spécimens « magnifiquement bien conservés » et ses relations hospitalières « admirables »; elle desservait bien sa région. Queen's obtenait à peine la note de passage : un nouveau laboratoire doté de méthodes « intelligentes » compensait des normes d'admission laxistes et de minces possibilités cliniques. Selon Flexner, Laval à Montréal (qui deviendrait éventuellement l'Université de Montréal) avait des normes d'admission « indéfinies », un équipement « médiocre » et de faibles liens avec l'université. La succursale de Québec était semblable, mais mieux reliée à son université. Les possibilités cliniques y étaient toutefois de « qualité limitée », à l'exception de l'abondance des cas en obstétrique.

Flexner réservait ses critiques les plus dures pour le Medical College de Halifax (N.-É.) et
Intérêts concurrents :

Aucun déclaré.

Cet article a été soumis à

l'examen des pairs.

Correspondance :

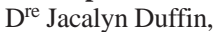
duffinj@queensu.ca

JAMC 2011. DOI:10.1503 /cmaj.110597

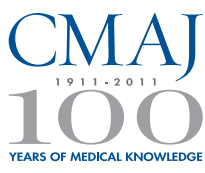

\section{POINTS À RETENIR}

- Le rapport influent publié en 1910 par Abraham Flexner au sujet de l'état des écoles de médecine en Amérique du Nord a créé tout un émoi chez les éducateurs médicaux au Canada.

- Les médecins du Canada se sont élevés contre les méthodes cavalières et les jugements abusifs de Flexner. Le rapport a néanmoins donné aux éducateurs médicaux du pays l'impulsion voulue pour entreprendre l'amélioration de leurs écoles.

- Le rapport Flexner a permis aux médecins de constater qu'une tribune de dialogue pancanadien s'imposait et il a par conséquent été un facteur précipitant de la création du JAMC en 1911. 
l'Université de Western Ontario à London (Ont.). Halifax « la faible » offrait une formation adéquate en sciences fondamentales, mais les nominations à l'hôpital étaient "faites par le gouvernement pour ses propres raisons », ce qui obligeait le Medical College à accorder le statut de professeur à des praticiens qui n'en étaient pas à la hauteur. Quant à western, ses normes d'admission étaient « dérisoires », ses installations cliniques "entièrement inadéquates », et ses laboratoires « misérables », tandis que les ouvrages à la bibliothèque étaient " gardés dans des casiers verrouillés » dont seul le concierge avait la clé. Il n'y avait aucun cours de physiologie, pharmacologie ou microscopie clinique, ni de dispensaire. La situation était, écrivit-il, « aussi mauvaise que ce que l'on peut trouver de ce côté-ci de la frontière ».

\section{Avec son premier numéro publié en janvier 1911, le JAMC entra en scène tambour battant.}

Flexner en concluait qu'aux moins trois des facultés canadiennes devraient fermer leurs portes. Aucune ne le fit.

Le 7 juillet 1910, au cours de la $57^{\mathrm{e}}$ assemblée de la Société médicale de la NouvelleÉcosse à Yarmouth, le D ${ }^{r}$ D.A. Campbell défendit l'école de Halifax et protesta contre la description « injuste » l'aiment qualifiée d'entité « commerciale ». Son discours fut publié à la fois dans le Maritime Medical News (juillet) ${ }^{8}$ et dans le Montreal Medical Journal (septembre) ${ }^{9}$, les deux journaux régionaux qui laisseraient la place à leur nouveau successeur national. Macphail quitta le Montreal Medical Journal pour devenir rédacteur en chef du JAMC, changement qu'il avait lui-même suggéré et qu'il attendait avec impatience depuis $1907^{10}$.

En décembre 1910, Albert Le Sage, rédacteur de l'Union Médicale du Canada, s'en prit avec virulence à Flexner, citant son «ineptie » et les « graves erreurs» qu'il avait disséminées si largement au sujet de Laval, mettant ainsi en danger le «prestige » d'une excellente école ${ }^{11}$. Le rédacteur se demandait pourquoi Flexner ne s'était pas donné la peine de lire les documents qui lui avaient été fournis et affirmait que c'était sans doute parce qu'il était « incapable de comprendre » des documents en français. Le Sage était également furieux contre Macphail, à qui il reprochait d'avoir réimprimé dans le Montreal Medical Journal les critiques injustes contre Laval en regard du rapport élogieux sur McGill ${ }^{11}$. La défense de la faculté de Laval fut ensuite prise en charge par le professeur de médecine L.E. Fortier ${ }^{12}$.

Avec son premier numéro publié en janvier 1911, le JAMC entra en scène tambour battant. Dans une envolée littéraire, Macphail expliqua la nécessité d'un journal médical pour répondre aux besoins médicaux du Canada. Il chercha à rassurer les journaux régionaux survivants en affirmant qu'il n'y aurait aucune concurrence $^{13}$. Pour faire preuve de solidarité devant cette unité médicale, un autre éditorial signala que les excellentes facultés de Toronto et de McGill avaient annoncé la réciprocité pour leurs étudiants, s'engageant à réaliser des échanges et espérant que les possibilités futures s'étendraient au reste du Canada et même jusqu'en Grande-Bretagne ${ }^{14}$.

Toujours dans ce premier numéro, des auteurs anonymes des facultés de médecine de Queen's et de Halifax protestaient contre les erreurs de Flexner et les sous-entendus dangereux contenus dans son rapport. Flexner n'avait passé « qu'une partie d'un après-midi » à la faculté de Queen's à Kingston et s'était trompé d'un facteur colossal de $6,{ }^{15}$ en comptant 80 lits cliniques.

L'auteur de Halifax s'y prit autrement ${ }^{16}$. Louant les bonnes intentions de Flexner qui souhaitait révéler des lacunes « incroyables » de facultés «peu solides », il reconnut les imperfections locales en les attribuant aux hostilités entre la faculté de médecine et l'Université Dalhousie. Il s'en prit toutefois lui aussi au rapport « biaisé, inexact et trompeur » de Flexner. Les laboratoires n'étaient pas «totalement misérables » et ne « sentaient pas mauvais». Flexner avait provoqué de l'étonnement et du ressentiment chez bien des diplômés exceptionnellement compétents de Halifax ainsi que chez leurs patients. Les étudiants fuiraient l'école ou la quitteraient. L'auteur prévenait que sans une intervention draconienne, cette campagne de dénigrement si largement diffusée pourrait entraîner la fermeture de l'école, ce qui réduirait encore davantage le nombre déjà insuffisant de médecins dans les Maritimes ${ }^{16}$.

Dans le numéro de mars 1911 du JAMC, un éditorial exigeait des changements à l'Hôpital de Halifax, notamment l'ajout de spécialistes et de résidences afin d'améliorer la formation et de faire avancer la science ${ }^{17}$. Le mois suivant, on faisait allusion une fois de plus au rapport Carnegie pour soutenir que toute "faculté de médecine devrait avoir son propre hôpital ${ }^{18} »$.

Toujours en avril, sous le titre " The vindication of Laval », le rédacteur du JAMC affirmait : «L'éducation médicale au Canada n'a rien à craindre des projecteurs les plus brillants qu'il est possible de braquer sur elle. S'ils révèlent des lacunes, on les corrigera. Nous ne souhaitons rien d'autre que le meilleur ${ }^{19}$. » Faisant allusion de 
façon sarcastique au privilège d'avoir fait l'objet d'une inspection officielle de la Fondation Carnegie, il décrivit la déception suscitée par un travail « effectué avec négligence ». Cet éditorial contenait la lettre d'excuses adressée par Flexner au doyen E.P. Lachapelle de Laval, à Montréal. Flexner y exprimait «son profond regret » d'avoir utilisé le mauvais catalogue pour évaluer l'école. (Flexner publia aussi une correction à cet effet dans le $J A M A^{20}$.) En terminant, Macphail affirmait : «Le rapport a été discrédité avec tant de justesse et à tellement d'égards qu'il est difficile de savoir quelle importance accorder aux éléments qui n'ont pas été contestés avec autant de succès ${ }^{19}$. »

Laval avait une bonne raison de se sentir vengée. Avec cet éditorial, Macphail s'excusait en fait de l'insulte qu'il avait adressée à Laval dans le Montreal Medical Journal. En retour, quelques semaines plus tard, Le Sage exhortait ses collègues francophones à adhérer à l'Association médicale canadienne pour son nouveau journal intéressant dont Macphail était le rédacteur « chevronné » et 《 cultivé $»^{21}$. La nation médicale s'unissait au-delà des frontières géographiques et linguistiques.

Dans le numéro d'octobre 1911 du JAMC, les facultés canadiennes décrivirent leurs améliorations et leurs plans pour le nouveau semestre ${ }^{22}$. On s'en prit encore au rapport Carnegie, « trompeur » par son caractère « injuste». Il reste que le sentiment d'urgence qu'il avait provoqué en Nouvelle-Écosse avait incité Dalhousie à venir « à la rescousse d'un établissement sérieusement handicapé », sauvant ainsi l'éducation médicale à Halifax pour la postérité et la région. L'école du Manitoba ajouta, elle aussi, son grain de sel, fière de la bonne note que lui avait attribuée Flexner mais osant avec insolence laisser entendre qu'il était allé trop loin en accordant davantage d'importance aux diagnostics en laboratoire qu' aux compétences au chevet. Enfin, Western se défendit en adoptant les propos mêmes de Flexner : « De ce côté-ci de la frontière, [...] aucune autre faculté de médecine du Dominion n'a fait l'objet d'autant de rapports injustes. [...] Ses ressources sont limitées [...] mais ses idéaux sont grands » - ce qui était l'évaluation même que Flexner avait faite de Johns Hopkins. «Au lieu de lutter contre le rapport », les professeurs de London avaient, toutefois, commencé « sur-lechamp à apporter des améliorations ${ }^{22}$. »

L'attitude cavalière de Flexner et ses jugements erronés sur les facultés de médecine canadiennes avaient certes provoqué du ressentiment, mais les écoles partageaient la vision pédagogique de Flexner et utilisèrent son rapport pour instaurer des changements souhaités depuis longtemps. En novembre 1911, le rédacteur du JAMC approuvait la disparition de la dernière école de marque au Canada (Halifax) ${ }^{23}$. Loin d'être une entreprise rentable, une bonne éducation en médecine était devenue une activité scientifique universitaire coûteuse. Ces mêmes idées furent reprises dans un rapport de suivi deux ans plus tard ${ }^{24}$.

Malgré des objectifs académiques communs, la profession médicale canadienne rejeta l'ingérence étrangère. Le Sage de Montréal avait été clair : "Vous êtes bien Américain M. ABRAHAM FLEXNER [...] à moins que vous ne soyez autre chose. [...] Pour nous, vous n'êtes qu'un bluffeur !"1 ». Macphail était du même avis, mais il poussa l'attaque personnelle au niveau du défi nationa ${ }^{25}$ :

Nous commençons à entendre parler de médecine américaine et de chirurgie américaine. [...] Le moment est venu de comprendre [...] que le Canada est de plus en plus différent des États-Unis [...] et des peuples européens dont il émane. Un type canadien de nationalité et un type canadien de maladie sont en train de prendre forme et les médecins du Canada jettent lentement, mais sûrement les assises d'une médecine canadienne de l'avenir. [...] Les enseignants [...] doivent faire tout leur possible pour former des hommes non seulement imbus de l'esprit scientifique, mais aussi bien dotés des connaissances nécessaires pour effectuer le travail extrêmement pratique qui est le leur.

Dans une allusion indirecte mais mordante à Flexner, il poursuivit ainsi : « Les enseignants cliniciens doivent toujours se souvenir qu'ils forment des hommes qui exerceront leur profession surtout dans des villes, dans des villages et à la campagne, loin des laboratoires bien équipés des hôpitaux et des universités. » Ils doivent apprendre aux étudiants à utiliser « les méthodes cliniques simples, mais éprouvées plutôt que de dépendre des diagnostics en laboratoire ». Il mentionna l'exemple des «skiagraphes» (images radiographiques) : merveilleux, bien entendu, mais inutiles «dans le bois, sur les rails » ou « dans un camp minier ». Il termina en lançant une demande de rapports cliniques : « Chaque personne qui contribue au journal peut aider à bâtir une médecine véritablement canadienne $^{25} \gg$. Quinze ans plus tard, Macphail se plaignait toujours des «méthodes américaines d'éducation en médecine ${ }^{26} »$.

Ce n'est pas par pure coïncidence que le centenaire du JAMC suit celui du rapport Flexner d'une seule année. Abraham Flexner avait uni les esprits et mobilisé les plumes des médecins et des éducateurs d'un bout à l'autre du Canada, les incitant à instaurer finalement un lieu de dialogue national. Ce n'était pas la première fois qu'un examen américain pousserait les Canadiens à se définir et s'exprimer par eux-mêmes. Ce ne serait pas non plus la dernière. 


\section{Références}

1. Shortt SED. Sir Andrew Macphail: physician, philosopher, founding editor of CMAJ. Can Med Assoc J 1978;118:323-6.

2. Flexner A. Medical education in the United States and Canada: a report to the Carnegie Foundation for the Advancement of Teaching, 1910; réimpression par facsimilé, New York (NY) Arno Press; 1972

3. Flexner A. I remember: the autobiography of Abraham Flexner. New York (NY) : Simon and Schuster; 1940; p. 128.

4. Ludmerer KM. Learning to heal: the development of American medical education. New York (NY) : Basic Books; 1985; p. 168-90.

5. Rothstein WG. American medical schools and the practice of medicine: a history. New York (NY) : Oxford University Press; 1987; p. 145-7.

6. Flexner A. I remember: the autobiography of Abraham Flexner. New York (NY) : Simon and Schuster; 1940; p. 121-2

7. Ludmerer KM. Learning to heal: the development of American medical education. New York (NY) : Basic Books; 1985; p. 172.

8. Campbell DA. Medical education in Nova Scotia. Maritime Medical News 1910;22:201-17.

9. Campbell DA. Medical education in Nova Scotia. Montr Med J 1910;39:628-49.

10. Robertson IR. Sir Andrew Macphail: the life and legacy of a Canadian man of letters. Montréal (Qc), Kingston (Ont.) : McGill-Queen's University Press; 2008 ; p. 47.

11. Le Sage A. Le bluff Américain : Fondation Carnegie et Université Laval à Montréal. Ignorance et préjugés. Union Med Can 1910;39:683-6.
12. Fortier LE. La Fondation Carnegie et l'Université Laval à Montréal. Union Med Can 1910;39:686-93.

13. Official notice. Can Med Assoc J 1911;1:57-8.

14. Reciprocity in teaching. Can Med Assoc J 1911;1:60-2.

15. Queen's and the Carnegie Report. Can Med Assoc J 1911;1:62-4.

16. Halifax Medical College. Can Med Assoc J1911;1:64-70.

17. Hospital troubles in Halifax. Can Med Assoc J 1911;1:254-8.

18. Medical school and hospital. Can Med Assoc J 1911;1:350-4.

19. The vindication of Laval. Can Med Assoc J 1911;1:354-6.

20. Flexner A. A correction of the Carnegie Foundation Report on Medical Education in Montréal. JAMA 1911;LVI:445

21. L'Association médicale canadienne. Union Med Can 1911;40:335.

22. Éditoriaux : Medical teaching in Halifax, Opening of the session, University of Toronto, McGill University, Manitoba Medical College, University of Laval at Montréal, Western University. Can Med Assoc J 1911;1:983-94.

23. The proprietary school. Can Med Assoc J 1911;1:1091-3.

24. Éditoriaux : The medical schools et On medical education. Can Med Assoc J 1913;3:977-96

25. Canadian medicine. Can Med Assoc J 1911;1:149-51.

26. Robertson IR. Sir Andrew Macphail: the life and legacy of a Canadian man of letters. Montréal (Qc), Kingston Ont.) : McGill-Queen's University Press, 2008; p. 237-9.

Affiliation : Chaire Hannah d'histoire de la médecine, Université Queen's, Kingston (Ont.).

Remerciements : L'auteure remercie Bert Hansen et Robert David Wolfe de leurs commentaires utiles. 